International Journal of Canadian Studies

Revue internationale d'études canadiennes

INTERNATIONAL JOURNAL OF CANADIAN STUDIES

REYUE INTERNATIONALE D'ÉTUDES CANADIENNES

\title{
Paysages, dépaysements. La construction de mythes identitaires dans l'art canadien moderne et contemporain
}

\section{Édith-Anne Pageot}

Numéro 36, 2007

Democracy, Rule of Law and Human Rights. Canada 25 Years after the Charter of Rights and Freedoms

La démocratie, l’État de droit et les droits de la personne. Le Canada, 25 ans après la Charte des droits et libertés

URI : https://id.erudit.org/iderudit/040786ar

DOI : https://doi.org/10.7202/040786ar

Aller au sommaire du numéro

\section{Éditeur(s)}

Conseil international d'études canadiennes

\section{ISSN}

1180-3991 (imprimé)

1923-5291 (numérique)

Découvrir la revue

Citer cet article

Pageot, É.-A. (2007). Paysages, dépaysements. La construction de mythes identitaires dans l'art canadien moderne et contemporain. International Journal of Canadian Studies / Revue internationale d'études canadiennes, (36), 287-305. https://doi.org/10.7202/040786ar
Résumé de l'article

Cet article s'intéresse aux rapports de l'art canadien, moderne et contemporain, à l'identité nationale. Il est un fait connu que les représentations picturales du paysage produites au Canada et au Québec entre 1910 et 1940 furent intimement liées aux nationalismes politiques. Palliant au traumatisme de la Première Guerre, les terres vierges dans la peinture du Groupe des sept furent associées à un nationalisme pancanadien, "viril » et salutaire. L'engouement pour la représentation de paysages ruraux chez les Québécois Clarence Gagnon, Marc-Aurèle de Foy Suzor-Coté et Marc-Aurèle Fortin firent plutôt écho au nationalisme d'un Lionel Groulx. À tort ou à raison, Paul-Émile Borduas incarna, quant à lui, les valeurs du nationalisme et de l'humanisme libéral qui caractérisèrent le Québec des années 1960. Aujourd'hui, le réfèrent identitaire national ne peut plus être considéré comme un réfèrent organisateur fort. Aussi, les performances de Rebecca Belmore et le travail de Lori Blondeau dénoncent l'eurocentrisme et les postulats phallocentriques que sous-tend l'histoire canadienne. Le déracinement, la dérive et l'exil traversent des pratiques diverses, celles de Christoper Pratt, de Rodney Graham, d'Isabelle Hayeur, de Camille Turner ou de Kinga Araya, entre autres. Ces entreprises de déconstruction n’ont pourtant pas mené à l'abandon d'une quête identitaire fondée sur l'appartenance à une communauté imaginée dans son rapport au " paysage ». Les images d'Isabelle Hayeur nient en même temps qu'elles l'éveillent le désir d'être en relation avec la nature. Et le travail récent d'Irène Whittome offre des images archétypes d'une communauté régionale dont l'identité collective se définit dans son rapport à la géologie du lieu.
Tous droits réservés @ Conseil international d'études canadiennes, 2007
Ce document est protégé par la loi sur le droit d'auteur. L'utilisation des services d'Érudit (y compris la reproduction) est assujettie à sa politique d'utilisation que vous pouvez consulter en ligne.

https://apropos.erudit.org/fr/usagers/politique-dutilisation/ 


\title{
Édith-Anne Pageot
}

\section{Paysages, dépaysements. La construction de mythes identitaires dans l'art canadien moderne et contemporain}

\author{
«(...) il est dans la nature de l'agent humain \\ d'exister dans un espace de questions concernant \\ les biens qu'il estime fortement, espace antérieur à \\ tout choix ou changement culturel contingent. " \\ Charles Taylor ${ }^{I}$
}

\section{Résumé}

Cet article s'intéresse aux rapports de l'art canadien, moderne et contemporain, à l'identité nationale. Il est un fait connu que les représentations picturales du paysage produites au Canada et au Québec entre1910 et 1940 furent intimement liées aux nationalismes politiques. Palliant au traumatisme de la Première Guerre, les terres vierges dans la peinture du Groupe des sept furent associées à un nationalisme pancanadien, " viril " et salutaire. L'engouement pour la représentation de paysages ruraux chez les Québécois Clarence Gagnon, Marc-Aurèle de Foy Suzor-Coté et Marc-Aurèle Fortin firent plutôt écho au nationalisme d'un Lionel Groulx. À tort ou à raison, Paul-Émile Borduas incarna, quant à lui, les valeurs du nationalisme et de l'humanisme libéral qui caractérisèrent le Québec des années 1960. Aujourd'hui, le référent identitaire national ne peut plus être considéré comme un référent organisateur fort. Aussi, les performances de Rebecca Belmore et le travail de Lori Blondeau dénoncent l'eurocentrisme et les postulats phallocentriques que sous-tend l'histoire canadienne. Le déracinement, la dérive et l'exil traversent des pratiques diverses, celles de Christoper Pratt, de Rodney Graham, d'Isabelle Hayeur, de Camille Turner ou de Kinga Araya, entre autres. Ces entreprises de déconstruction n'ont pourtant pas mené à l'abandon d'une quête identitaire fondée sur l'appartenance à une communauté imaginée dans son rapport au " paysage ". Les images d'Isabelle Hayeur nient en même temps qu'elles l'éveillent le désir d'être en relation avec la nature. Et le travail récent $d$ 'Irène Whittome offre des images archétypes d'une communauté régionale dont l'identité collective se définit dans son rapport à la géologie du lieu.

\begin{abstract}
This article looks at the connections between modern and contemporary Canadian art and national identity. It is a known fact that, between 1910 and 1940, pictorial representations of landscapes produced in Canada and in Québec were intimately linked to politics of nationalism. Disguising the traumas of the First World War, virgin lands in the work of the Group of Seven
\end{abstract}


were associated to a pan-Canadian nationalism - "virile" and salutary. For Québécois like Clarence Gagnon, Marc-Aurèle de Foy Suzor-Coté and Marc-Aurèle Fortin, infatuation with representations of rural landscapes rather echoed the nationalism of Lionel Groulx. And rightly or wrongly, Paul-Emile Borduas, for his part, incarnated the values of nationalism and liberal humanism that characterized Québec in the 1960s. Today, the national identity referent can no longer be considered as a strong organizing referent. Also, the performances of Rebecca Belmore and the work of Lori Blondeau denounce Eurocentrism and the phallocentric premises that underlie Canadian history. The uprooting, the drifting and the exile run through diverse practices-those of Christopher Pratt, Rodney Graham, Isabelle Hayeur, Camille Turner or Kinga Araya among others. Yet, these enterprises of deconstruction did not lead to an abandonment of the quest for identity -one founded on belonging to an imagined community in its relation to the "landscape." The images of Isabelle Hayeur both deny and awaken the desire for a connection with nature. And the recent work of Irène Whittome offers archetypal images of a regional community whose collective identity is defined in its relationship to the geology of place.

Les problèmes actuels liés à l'utilisation abusive des ressources naturelles et à la détérioration des flores et des faunes terrestre et aquatique ont amené plusieurs artistes canadiens à réfléchir sur notre rapport à l'environnement naturel. Pensons entre autres à Jean-Pierre Aubé, Edward Burtynsky, Isabelle Hayeur, Paul Hess, Kévin Schmidt, mais aussi à la photographe Lorraine Gilbert et aux peintres Wanda Koop, Stephen Hutchings et Eleonor Bond ${ }^{2}$. L'installation récente d'Aubé, intitulée Save the Waves (2004), vise par exemple à transformer des phénomènes naturels en paysages sonores. Aubé a mis au point des dispositifs qui lui permettent de capter et de rendre audibles les ondes VLF (Very Low Frequencies) qui proviennent de la magnétosphère. Or actuellement, même en régions éloignées, ces basses fréquences tendent à être submergées par les systèmes de télécommunication militaires et commerciaux. Dans ce contexte, les installations d'Aubé forment un répertoire de phénomènes naturels menacés de disparition, mais elles agissent aussi à titre d'amplificateur de la pollution électromagnétique provenant du milieu ambiant. Les projets d'Aubé constituent donc en quelque sorte une écologie des ondes. Les photographies du Torontois Burtynsky dénoncent, quantà elles, les ravages écologiques du capitalisme global en Chine, au Bangladesh, en Italie, au Vermont et ailleurs. Les montages numériques de l'artiste montréalaise Isabelle Hayeur explorent les sentiments d'aliénation et de dislocation vécus dans les banlieues urbaines. Paul Hess, un artiste travaillant dans la région de Vancouver, table sur l'idée de frontière, d'effondrement et d'affaissement du terrain en lien avec la perte d'identité et le sentiment d'incertitude. Et le jeune Kévin Schmidt s'intéresse aux codes visuels et aux clichés typiques des paysages pittoresques façonnés par l'industrie touristique. 
Bien que distinctes dans leurs démarches et dans leurs intentions, plusieurs pratiques artistiques contemporaines partagent à l'évidence un intérêt commun pour le paysage. Et en dépit des nuances qui s'imposent, ces œuvres contribuent à faire resurgir ce thème dans l'art canadien. Il va sans dire que le paysage est envisagé ici dans une perspective large, c'est-à-dire en tant que lieu d'occupation humaine ${ }^{3}$ nouant la vie culturelle à la vie sociale, à l'espace géographique, au territoire et au climat, et non pas seulement en tant que représentation de la nature environnante. C'est dire que le paysage est aussi un lieu de façonnement de l'identité. À ce sujet, rappelons que les représentations du paysage dans la peinture moderne au Canada avaient joué un rôle capital dans la construction d'une identité collective liée à la nationalité. Délibérément ou non, plusieurs artistes, en particulier des peintres, avaient fourni au moyen du paysage un sens d'appartenance nationale fondé sur la location géographique, ses particularités réelles ou mythiques. Déclassé par l'art abstrait puis bientôt par le pop art, l'op art et enfin l'art conceptuel, le paysage comme genre pictural cessera graduellement d'occuper une position dominante dans l'art canadien.

La résurgence des références à la nature dans l'art contemporain permet de repenser et de réactualiser le rapport de l'art au paysage. À travers des pratiques artistiques actuelles choisies, cet essai se penche donc sur ce renouveau du paysage. L'idée de retour, ou plus précisément de renouveau, suppose une lecture qui s'inscrit en dialogue avec une tradition passée récente. Nous voulons tenter de caractériser l'utilisation de certaines références au paysage dans l'art actuel «post-moderne", mais aussi tenter de situer cette pratique par rapport à un passé récent qui campe le paysage dans un rapport complexe aux idéologies politiques. Maintenant que la dissolution des visions unificatrices du paysage va de pair avec la perte de référent identitaire collectif organisateur, quelle est la nature du rapport de l'art au paysage?

On sait bien que dans l'art moderne canadien, le paysage fut un lieu privilégié de construction identitaire. Certes, ce lien étroit entre la nationalité et le paysage ne fut jamais exclusif à l'art moderne, ni au Canada $^{4}$. Il reste qu'entre 1910 et la fin des années 1930 , le paysage était un thème pictural particulièrement dominant à Toronto et à Montréal. Est-il besoin de rappeler que la vision du paysage qu'avait promue le Groupe des sept (1920-1931) - formé des peintres Tom Thompson, Lawren Harris, A. Y. Jackson, Frank Carmichael, J. E. H. MacDonald, Arthur Lismer et Fred Varley - fut célébrée à la manière d'un véritable emblème national s'immisçant jusque dans la culture populaire par l'entremise de cartes postales, de calendriers et de brochures touristiques? Ces images ont fait plus que fournir à l'imaginaire collectif un espace naturel idéal, elles ont véritablement affermi les sentiments d'identité nationale, surtout au Canada anglais. Au Québec au cours de la même période, les tableaux des 
peintres dits régionalistes alimentaient quant à eux une autre version de la nationalité plus proche des discours politiques traditionalistes attachés aux valeurs du passé.

Comme nous le rappelons dans la première partie de cet essai, plusieurs mouvements canoniques de l'art moderne au Canada furent envisagés à partir de ce lien, présumé ou réel, du paysage à la nationalité. Qui plus est, ni les uns ni les autres n'échappèrent à l'emprise d'un discours politique polarisé autour des problématiques liées aux nationalismes, et ce, en dépit des intentions profondes et parfois divergentes qui alimentaient leur pratique artistique respective. Leur amarrage à la nationalité, réel ou fantasmé, a débouché sur l'édification de véritables mythes fondateurs en lien avec diverses positions nationalistes débattues dans l'arène politique. Par exemple, l'art du Groupe des sept, celui des peintres régionalistes québécois et même l'art pourtant abstrait de Paul-Emile Borduas furent perçus à tort ou à raison comme étant imprégnés ou pouvant évoquer des positions nationalistes diverses.

\section{Le paysage en tant que territoires politiques}

Malgré des débuts difficiles dans un milieu plutôt hostile à l'art moderne, les œuvres des Sept incarnaient dès la fondation du groupe un nationalisme " viril » et pancanadien, qui sera érigé quelques années plus tard en une authentique mystique canadienne ${ }^{5}$. En fait, à son retour de GrandeBretagne en 1908, Lawren Harris avait déjà la conviction profonde que l'unité du peuple canadien devait s'enraciner dans la référence au paysage nordique ${ }^{6}$. Dans l'avant-propos qu'il rédigea lors de la première exposition du Groupe présentée à l'Art Gallery of Toronto 16 ans plus tard, soit en mai 1920, Lawren Harris exhortait le public au patriotisme : "Aucun pays ne peut espérer s'élever au-dessus du vulgaire s'il n'accorde à ses citoyens une confiance illimitée (...) Un peuple doutant d'égaler ou de surpasser la stature de quelque prédécesseur se devrait d'émigrer au service d'une civilisation supérieure ${ }^{7}$ ". Les Sept proposaient une vision épurée du paysage, une vision qui ignorait les spécificités culturelles valorisant la magnificence du Nord ${ }^{8}$ et du bouclier canadien. Palliant le traumatisme de la Première Guerre mondiale, ces paysages sauvages, ces terres vierges faisant table rase de la civilisation et de la différence apparaissaient sans aucun doute salutaires'. Si bien qu'en 1925, cette vision du paysage demeurait aux yeux du critique d'art Newton MacTavish l'enjeu le plus important de l'art canadien. Selon lui, seuls les paysages des Sept fournissaient " veritable interpretations of national characteristics ${ }^{10}$." Fondant son plaidoyer sur l'existence d'un rapport irréductible entre l'art et le territoire, le critique Fred Housser défendait, lui aussi, l'esthétique du groupe dans son livre intitulé $A$ Canadian Art Movement: The Story of The Group Of Seven ${ }^{11}$ en avançant l'idée selon laquelle un art national authentique ne pouvait exister que dans cette forme privilégiée de paysage. Ces conceptions liant le paysage intact et à conquérir à la définition d'un 
tempérament viril et proprement canadien ont marqué l'histoire de l'art moderne au Canada au point de perdurer pendant plusieurs décennies. Signalons à ce propos que c'est bien par référence au Groupe des sept que la commissaire Elizabeth Wynn Wood homologuait le travail des artistes canadiennes qu'elle présentait à New York pour la première fois en $1947^{12}$.

Le paysage fut également un motif privilégié dans l'élaboration d'une spécificité " canadienne-française ", antithèse d'un nationalisme pancanadien aux aspirations universelles. François-Marc Gagnon ${ }^{13}$ a montré qu'à l'opposé du regard décanté des Sept, certains artistes québécois ont adhéré à un régionalisme pictural fondé sur la mise en valeur de la vie rurale ou de la forêt en choisissant pour ce faire les Laurentides, l'île d'Orléans ou la région de Charlevoix, par exemple. L'engouement pour la représentation de paysages ruraux, agricoles et partagés en lots, chez Clarence Gagnon, Marc-Aurèle de Foy Suzor-Coté et Marc-Aurèle Fortin pour ne citer qu'eux, pouvait en quelque sorte faire écho à un nationalisme traditionaliste qui croyait à l'urgence de conserver un héritage français paysan fondé sur la famille, la paroisse et la vie rurale. Aussi, les scènes rurales et les représentations du travail de la terre pouvaient aisément se trouver associées à la recherche d'authenticité d'une « race canadienne-française " caractérisée par l'attachement au terroir et à l'héritage français telle que la défendaient un Henri Bourassa au Devoir ou un Lionel Groulx à $L$ 'Action française. Certes, le paysage rural ne fut pas un thème exclusif à ces artistes. Â titre d'exemples, on se rappellera que Suzor-Côté a aussi exploré la voie de la peinture d'histoire, le nu et le portrait. Fortin, quant à lui, a peint des paysages urbains, des vues du quartier Hochelaga et des scènes du port de Montréal, sujets qui témoignent d'une réflexion sur le rapport entre culture et nature. Il reste que les représentations de la nature peintes dans Arthabaska par Suzor-Côté participent à la construction d'un art national ${ }^{14}$. Et plusieurs cuvres de Fortin idéalisent la culture traditionnelle. L'espace réservé aux immenses arbres dans ses paysages urbains des années 1920 et la récurrence de la charrette de foin dans ses paysages de la vie rurale réédifient certaines des valeurs fondamentales de l'idéologie clérico-nationaliste, soit la valorisation de l'agriculture et de la ruralité. En outre, Fortin est bien connu comme un défenseur de la culture traditionnelle et de sa mise en valeur patrimoniale. En témoigne cette lettre où il dénonce l'inaptitude des habitants à repérer les éléments architecturaux régionaux qui ont, selon lui, une valeur identitaire séculaire :

Les populations rurales actuelles n'ont aucun goût artistique; elles sont d'une ignorance crasse et ne comprennent pas que ce qui fait le charme d'un village, ce qui lui donne son caractère et qui attire les visiteurs ce sont ces antiques demeures construites par les ancêtres. Alors au lieu de les conserver pieusement, de les garder telles qu'elles sont, on s'efforce de les rajeunir, de leur donner une apparence moderne ${ }^{15}$. 
Au cours des années 1940, Paul-Émile Borduas, le chef de file des Automatistes, verra à développer un vocabulaire abstrait qui visait à affranchir l'art du curriculum académique et de toutes visées idéologiques fussent-elles politiques ou religieuses. Il importe toutefois de ne pas perdre de vue que l'importante figure de Paul-Émile Borduas et le célèbre manifeste Refus global (paru en 1948), dont il avait été l'instigateur, incarneront aux yeux de plusieurs les idéaux politiques de la Révolution tranquille. Certes, la majorité des signataires du manifeste, en tout premier lieu Borduas lui-même, étaient peu engagés dans l'action politique. Dans une lettre à Claude Gauvreau, Borduas déclarait à la fin de sa vie : « J'ai en horreur tout nationalisme. Je reste apolitique ${ }^{16}$. $\gg$ Il n'en demeure pas moins que le texte de Refus global et sa fortune critique sont politiques. Appel à la catharsis, le célèbre pamphlet dénonçait les sentiments de honte de soi liés à l'état pathologique de la névrose identitaire des francophones québécois, "petitpeuple »colonisé et catéchisé. Ce constat mettait de l'avantl'urgence et la nécessité d'une véritable cure psychanalytique de la conscience collective des francophones en terre québécoise, qui s'avouaient bafoués par la colonisation et contaminés par l'endoctrinement religieux : « La honte du servage sans espoir fait place à la fierté d'une liberté possible à conquérir de haute lutte. Au diable le goupillon et la tuque ${ }^{17}$ ! $»$. Il ne faudrait pas en conclure qu'une telle critique de la société québécoise trouvait un équivalent sur le plan esthétique; il ne fut jamais question d'une transposition franche ou d'une assimilation univoque. Nous nous rendons à l'idée que l'abstraction gestuelle qu'avaient développée Borduas et ses collègues du groupe automatiste, et bientôt post-automatiste, répondait au besoin de repérer et de définir l'avènement d'une modernité artistique non figurative qui répondait à leur exploration de l'inconscient et qui s'inscrivait en rupture avec la tradition académique. À ce sujet, la relative marginalisation d'Alfred Pellan dans le panthéon des grands artistes québécois est éloquente. Au-delà de cette exigence, l'art abstrait des Automatistes collait également au désir de libération totale exprimé dans le manifeste Refus global. Conséquemment, nous avons bien de la peine à ne pas penser que l'affranchissement total sollicité par le manifeste fut mis en cuvre par l'abstraction gestuelle.

À tout prendre, il ne faut pas sous-estimer la présence d'une charge critique ainsi que la référence implicite au paysage qu'entretiennent les abstractions de Borduas réalisées après la parution de Refus global. On se rappellera que la sortie du manifeste avait entraîné immédiatement la miseà pied de Borduas à l'École du Meuble ainsi que l'interdiction pour lui d'enseigner dans les écoles de la province. L'isolement, la précarité financière et les difficultés familiales qui accablaient Borduas dans les années qui ont suivi cet épisode le conduiront graduellement à envisager $l^{\prime}$ exil ${ }^{18}$. Au cours des années 1950, Borduas quitte donc le Canada vers les États-Unis ${ }^{19}$. De Boston, il se rend à Provincetown au printemps 1953 et ensuite. $\mathrm{New}$ York $^{20}$. Dans ce contexte, cen'est pas un hasard si les thèmes 
de résistance, d'enfermement, d'ouverture et d'accueil hantent les cuvres abstraites de 1953 et de 1954 . Pensons par exemple à : Réception automnale, Résistance végétale, L'étang recouvert de givre, Les Pins incendiés, Plante généreuse, toutes de 1954.

Davantage intéressé à sonder les territoires de l'inconscient, Borduas ne s'intéresse pas vraiment au paysage comme genre pictural. Notons toutefois que les titres attribués à cette production du début des années 1950 ainsi que la gamme de couleurs utilisées ne désavouent aucunement la référence au paysage, tout comme les tableaux réalisés en 1953 : Neige d'octobre, La danse sur le glacier, Les coups de fouet de la rafale, pour ne nommer que ceux-là ${ }^{21}$. La critique, tant canadienne qu'américaine, n'avait d'ailleurs pas manqué de souligner cet aspect. Encore en 1957, à propos d'œuvres abstraites comme 3+4+1 (1956) ou Pulsation (1955), dont les titres ne justifient pourtant pas la métaphore paysagiste, le critique américain Carlyle Burrows arguait que :

Il se pourrait bien que la beauté de l'hiver canadien ait exercé quelque influence de nature sentimentale sur le peintre Paul-Émile Borduas, dont les derniers tableaux sont exposés présentement chez Martha Jackson. La plupart de ceux-ci, en effet, évoquent par la blancheur sensible de la surface de la toile l'atmosphère décolorée des étendues enneigées, et cette impression est accentuée par le traitement de l'œuvre, qui est parfois maculée et habilement maçonnée pour créer des effets de grande turbulence ${ }^{22}$.

Il est certain qu'au Canada, les paysages d'hiver et la neige plus particulièrement ont nourri l'imaginaire collectif. Certains chercheurs, dont Suzanne Labrie ${ }^{23}$, vont même jusqu'à suggérer la pertinence d'un lien étroit entre la rigueur des hivers canadiens et certaines caractéristiques psychologiques telles que la détermination et l'adaptabilité. Ces idées ne sont pas récentes. Déjà au XIX ${ }^{\mathrm{e}}$ siècle, le père François-Edné Rameau soutenait l'idée d'une filiation entre la nationalité, ses caractéristiques raciales et le climat, en l'occurrence l'hiver ${ }^{24}$. La neige fait donc partie d'un répertoire d'images qui a profondément marqué l'imaginaire collectif canadien. C'est dire que même en l'absence d'indice concernant un lieu géographique circonscrit, comme c'est le cas avec la peinture abstraite de Borduas, qui se voulait avant tout une peinture de signes, les représentations évoquant l'hiver par le titre, le format ou la palette pouvaient facilement être rabattues par la critique sur des questions de spécificité nationale ${ }^{25}$. D'ailleurs, c'est également la raison pour laquelle les performances exécutées dans la neige (Snow White Series: Knowing the end is near (2000) ou White Water I (2001) entre autres) par la jeune Tanya Kitchell, une artiste de Toronto, sont actuellement célébrées en Europe. Le désir de l'artiste de faire corps avec la neige est perçu comme un aspect pittoresque de son travail. Une interprétation qu'elle conteste fermement ${ }^{26}$. 
D'une part, Borduas ainsi que le célèbre Refus global personnifieront à partir du milieu des années 1950 et davantage au cours des années 1960 les aspirations à la liberté sous-jacente à la question nationale au Québec. Dans ses Mémoires, Georges-Émile Lapalme, alors ministre des Affaires culturelles, fait de Borduas une figure héroïque qui justifie dans une certaine mesure la lecture politique qu'il faisait de l'art moderne au tournant des années $1960:$ «J'avais toujours considéré comme pitoyable la place que l'État avait réservée aux artistes, particulièrement aux peintres. Le sort fait à Borduas m'avait paru inique 27 . " D'autre part, la persistance des références au paysage donne paradoxalement au travail abstrait de Borduas un ancrage possible dans la nationalité. Il faut dire qu'au cours des années 1960, la nation pouvait encore être un référent identitaire structurant capable de réguler d'autres référents tels que l'appartenance de classes, l'ethnie, l'identité sexuelle ou le positionnement générationnel, entre autres.

Au-delà de ces considérations et de la nostalgie un peu naïve exprimée par la critique à propos des œuvres de Borduas, en quoi la référence récurrente au paysage est-elle significative? Il est permis de penser que les métaphores évoquant le paysage expriment la tension, l'aliénation, mais aussi l'espoir qu'entraînait la migration de Borduas. Dans cette perspective, c'est-à-dire en lien avec sa condition d'exil, on conviendra de l'importance de la quête identitaire sous-jacente à ce travail du début des années 1950. Sur ce point, Louise Vigneault a interprété la production tardive de Borduas, soit celle de la fin des années 1950, où s'opposent les valeurs de noir et de blanc, comme l'expression d'une synthèse identitaire entre un sentiment d'appartenance à la culture canadienne-française et un sentiment d'appartenance à l'Amérique du Nord ${ }^{28}$. Notre hypothèse porte plus spécifiquement sur les abstractions produites entre 1953 et 1956 parmi lesquelles subsiste une allusion au paysage. De façon récurrente, Borduas oscille entre un choix de titres, une palette et une touche qui suggèrent soit l'éclatement de l'horizon soit un espace figé, et dont les thèmes évoquent la résistance ou l'accueil. À l'opposé d'une synthèse identitaire, Borduas paraît ici mû par une dichotomie : un désir de débordement des frontières s'exprimant à la fois par l'éclatement des formes figuratives et des plans et par un besoin d'enracinement dans une communauté d'être spécifique. Quoi qu'il en soit, c'est bien au moyen d'évocations du paysage que Borduas réfléchit sur la quête identitaire et les contradictions qui l'animent. Ce n'est pas un hasard si à la veille de sa mort, en 1960, Borduas reprend la métaphore paysagiste pour exprimer ce qu'il estime être l'essentiel de sa pratique : " Au fond, l'élément de ce monde qui me demeure le plus permanent, le seul peut-être, c'est la peinture, la peinture physique, la matière, la pâte. C'est là mon sol natal, c'est ma terre. Sans elle je suis déraciné. Avec elle, que je sois à Paris ou ailleurs, peu importe - je suis chez moi ${ }^{29}$. " Malgré la propension à l'universalisme dont se réclame l'artiste à la fin de sa vie, le paysage, à la manière d'un stigmate, continue de 
hanter sa quête identitaire. La quête des origines, d'un espace originel, d'un état initial fantasmé s'exprime chez lui par un lien privilégié à la terre.

\section{Déracinement}

Maintenant que les notions de mouvance et de pluralité réfutent l'idée jadis opérante d'un référent identitaire organisateur, tel que la nationalité, comment les artistes canadiens en arts visuels, ceux qui continuent de s'intéresser au paysage, négocient-ils le rapport de l'art à son contexte d'émergence? La nationalité n'est plus, aujourd'hui, un référent identitaire collectif fort. Multiple, évolutive, dynamique et en devenir, l'identité ne peut plus être emmurée dans la nationalité ou les nationalismes tous azimuts. Comme le rappelle Jocelyn Maclure : « Il faut cesser de voir la nation comme, d'un côté, la seule ou nécessairement la plus importante source d'identification collective et, de l'autre, comme un lieu d'incarcération identitaire ${ }^{30}$. $\gg$ Pourtant, cela ne contrevient pas au fait que la nationalité continue de façonner l'identité collective, en témoignent les luttes menées en faveur d'une certaine autonomie politique et réclamant la reconnaissance de la diversité culturelle ${ }^{31}$. La violence urbaine qui a sévi dans les banlieues françaises à l'automne 2005 montre à quel point l'expérience transculturelle post-moderne, celle du citoyen mondialisé, ne correspond pas au vécu de tous ni même à celui de la majorité. C'est dire que le rapport des individus au territoire qu'ils habitent continue d'être un élément important de réflexion jouant un rôle déterminant dans le sentiment d'appartenance à un groupe. Aussi, plusieurs pratiques artistiques contemporaines sont-elles traversées d'une quête identitaire fondée sur l'appartenance à une communauté ethnique en rapport avec le territoire qu'elle occupe. Parmi elles, il convient de citer le travail de Rebecca Belmore et de Lori Blondeau, dont les performances et les installations transgressives explorent la réalité des communautés aborigènes en dénonçant l'eurocentrisme et les postulats phallocentriques qui sous-tendent l'histoire canadienne. Sa performance Fountain (2005) en est un bon exemple. La vidéo de cette performance, filmée par Noam Gonik, s'ouvre sur une vue panoramique de l'océan à partir de Iona Beach. On y voit Belmore lutter pour extirper des eaux un seau qu'elle transporte laborieusement sur le rivage. Ce combat évoque métaphoriquement l'arrivée des Européens sur le continent; les flots houleux incarnent le traumatisme culturel et les bouleversements écologiques conséquents. Belmore soulève ensuite péniblement le seau dont elle déverse le contenu en éclaboussant délibérément la camera : il ne s'agit pas d'eau mais de sang, le sang de tous les malheureux engagés dans une lutte pour le pouvoir et la possession des terres. Le geste se veut une métaphore du fardeau imposé aux Premières nations par l'action colonisatrice des Européens. Animées d'une charge critique analogue, les interventions de Lori Blondeau, une artiste originaire de Regina, parodient la culture continentale américaine. La boîte lumineuse intitulée Cosmosquaw (1996) représente une cow-girl autochtone vêtue et maquillée à la manière des cover-girls américaines. 
Quant à sa performance $A$ Moment in the Life of Belle Sauvage (2002), elle met en scène le personnage Belle Sauvage héritière d'un passé familial autochtone imprégné de culture western. Le grand-père de Blondeau était un cow-boy indien, explique-t-elle. Caressant un désir d'autonomie, sa lecture postcoloniale de l'histoire canadienne vise à exposer l'effacement, l'aliénation et les traumatismes de la culture autochtone. Mais elle tente aussi d'aménager un espace à partir duquel on pourrait imaginer une identité plurielle, un espace où pourraient coexister les multiples manières nationales d'être en Amérique.

D'autres artistes travaillent l'idée de mutabilité, d'hybridité ainsi que les sentiments de déracinement et d'exil liés soit à une diaspora ethnique soit à l'émigration. Je pense respectivement au projet Miss Canadiana (2004) de la jeune Torontoise d'origine jamaïcaine Camille Turner et au travail de la Montréalaise d'origine polonaise Kingá Araya. Dans PolCan (2003), mot hybride se référant à la Pologne et au Canada, Araya était chaussée de deux hémisphères dont les talons arrondis rendaient la marche instable, étourdissante, branlante. La quête identitaire qui traverse l'œuvre se présentait comme autant de métaphores de situations d'échec et d'incertitude. Ces situations de faillite en lien avec le dépaysement relatifà l'émigration mettent en scène un sujet incertain et nomade au sens où l'entend la pensée poststructuraliste, c'est-à-dire constamment aliéné, une identité instable, voire traumatisée, dans un corps anarchique ${ }^{32}$. D'ores et déjà, nous savons que de telles démarches fragilisent, remettent en question et déconstruisent la référence identitaire nationale. Il nous reste à nous pencher sur des démarches qui incluent des représentations du paysage et travaillent plus spécifiquement à partir de ce motif.

Indubitablement, les pratiques artistiques actuelles qui perpétuent l'intérêt des modernes pour le paysage entretiennent un rapport dialogique avec un imaginaire collectif profondément imprégné de liens présumés à la nationalité. Il est nécessaire de préciser que c'est sous le mode de la subversion que plusieurs artistes lient le paysage à la formation de l'identité. Mentionnons à titre d'exemple, l'œuvre Camera Obscura (1979) de l'artiste conceptuel Rodney Graham. Cette installation bâtie près d'Abbotsford, C.-B., consistait en une énorme camera obscura dans laquelle le spectateur était invité à entrer pour observer l'image d'un arbre interverti. Il s'agissait de faire vivre au visiteur l'expérience du paysage, si cruciale au sein de la culture canadienne, à travers un processus de médiation qui rend caduque l'idée romantique du paysage sauvage. Avec des moyens divers, une telle entreprise de déconstruction et de démystification de l'histoire canadienne au moyen d'une utilisation subversive du paysage traverse également la production d'autres artistes tels que Paul Hess, Don Gill, Greg Hill, Kent Monkman, Christopher Pratt pour ne nommer qu'eux. On pourrait multiplier les exemples, attachons- 
nous plutôt au travail de ce dernier puisqu'il fait aujourd'hui partie des artistes célébrés dans le milieu des arts visuels au Canada.

À l'automne 2005, le Musée des beaux-arts du Canada à Ottawa consacrait au Terre-Neuvien Christopher Pratt une grande exposition rétrospective. L'exposition ancrait le travail du peintre dans un rapport inéluctable à la nationalité et à la mise en valeur d'une spécificité canadienne liée au territoire. À cette fin, le texte d'introduction reproduit sur les murs d'entrée rapportait ce commentaire du mécène I. David Marshall : « Christopher Pratt is one of the country's best forever living artists capting the Canadian soul (...) ». À vrai dire, l'appréciation du mécène venait répudier le contenu agitateur du travail et l'idée d'une crise identitaire sous-jacente à la peinture de Pratt. L'artiste s'est toujours avoué touché par les traumatismes identitaires qui ont résulté de l'annexion de Terre-Neuve à la Confédération canadienne le $1^{\text {er }}$ avril 1949. On admet par conséquent la teneur politique de son engagement. Sous ce rapport, l'expérience du lieu ainsi que les notions structurantes de vide, d'ouverture, d'enfermement et de déséquilibre dans son travail des 20 dernières années s'inscrivent dans une critique des politiques colonialistes du gouvernement fédéral.

Les motifs iconographiques ainsi que les moyens formels mis en oeuvre par Christopher Pratt évoquent clairement l'idée d'une communauté dépouillée de ses récits identitaires. Les thèmes picturaux qu'il privilégie comprennent autant d'embrasures bouchées, d'espaces architecturaux évacués, de cadrages déphasés que de fenêtres tronquées. La gamme chromatique finement calculée, jamais franche ou contrastée, situe la scène à un moment de la journée difficile à préciser, un moment de transition entre le jour et la nuit, ou un point de rupture entre le début ou la fin d'une tempête de neige. À cet égard, les scènes d'hiver de 2004 représentant le port de Whiteway sont probantes. À une architecture abandonnée vue en raccourci se juxtapose un espace temporel flou conférant à la scène des sentiments de dislocation et de détournement. Par conséquent, l'élément spatiotemporel chez Pratt intéresse davantage l'absence de finitude que la construction d'un espace structurant. Et la réflexion identitaire sous-jacente à son travail évoque la dispersion plutôt que la définition d'une âme canadienne. C'est donc artificiellement que le commentaire antinomique du mécène, cité plus haut, attribue une unité signifiante à une communauté disloquée.

\section{Dérives}

$\grave{A}$ partir du paysage, d'autres artistes, plus jeunes cette fois, centrent également leur démarche sur l'idée de débordement et sur l'absence de finitude en créant des espaces incertains et difficilement configurables. Outre cette caractéristique, les œuvres empruntent souvent les apparences du paysage panoramique issu du $\mathrm{XIX}^{\mathrm{e}}$ siècle. C'est le cas de certaines œuvres des artistes montréalais Ivan Binet, Bertrand Rainville Pitt et 


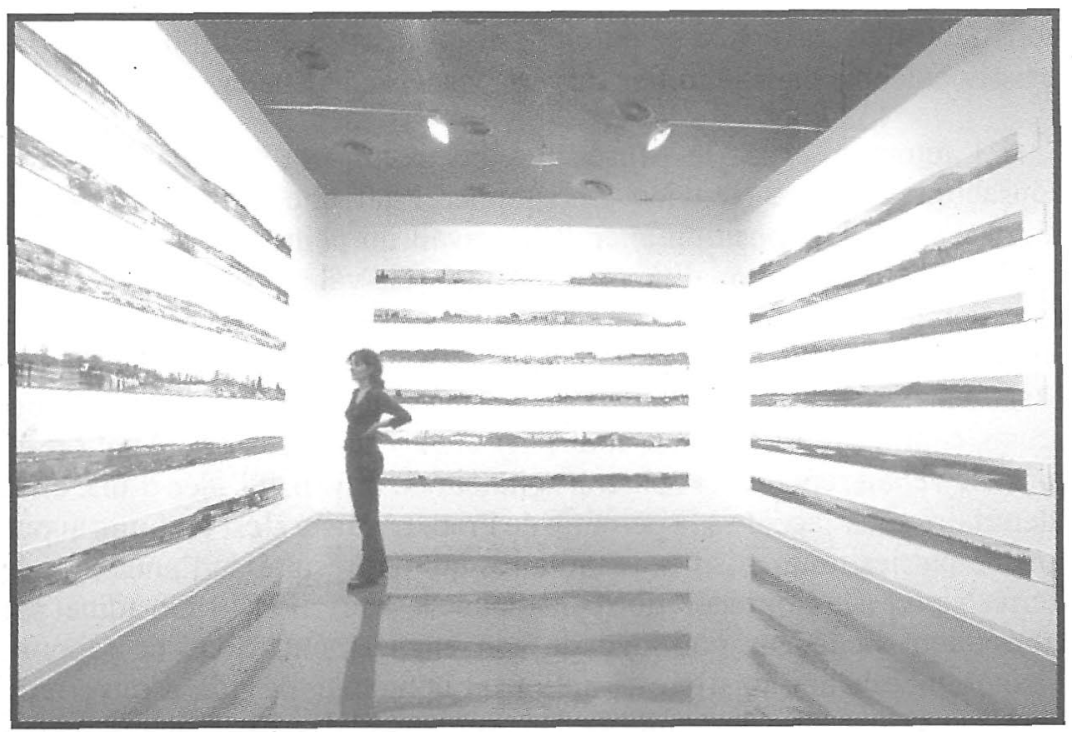

\section{Figure 1}

Ivan Binet, Répertoire d'horizons, 2002. Galerie Vox. Gracieuseté de l'artiste

Isabelle Hayeur. L'œuvre photographique en noir et blanc d'Ivan Binet intitulée Répertoire d'horizons (2002, fig.1) se déploie sur des bandes horizontales continues. Le format épouse littéralement la ligne d'horizon obligeant un balayage visuel en une sorte de préhension totale de l'espace. Ici, le répertoire semble contrevenir au parti pris du morcellement et de toute vulnérabilité inquiétante. Or, par définition, répertorier consiste à établir une nomenclature fondée sur des règles et des cadres, ce qui présuppose la maîtrise de l'objet d'études. Pourtant, il est intéressant de signaler qu'un examen plus minutieux des images, en apparence très réalistes, révèle le côtoiement d'éléments antinomiques et d'espaces géographiquement incongrus. Si bien qu'au fond, il est plutôt question d'excès et de débordement que d'entièreté et de finitude.

Entre 2000-2002, Bertrand Rainville Pitt a réalisé une installation vidéo sonore placée sous le thème de la Dérive (fig. 2). Un grand écran lumineux projette des images fluctuantes d'un paysage filmé en Suisse à l'occasion d'une résidence dans les studios de la Fondation Christoph-Merian. Des images de nappe d'eau, des vues du ciel défilent lentement sur l'écran lumineux. Ces images se reflètent, inversées, dans un bassin posé au sol, rempli d'eau et agissant comme un miroir. L'installation visuelle est accompagnée d'un échantillon sonore enregistré en synchronie avec la séquence des images. Par l'amplification des basses fréquences, l'artiste visait à rendre audibles les bruits de « silence, (...) les bruits sourds et profonds ${ }^{33}$ », les murmures du cœur, les frémissements de la terre. Brouillé, superposé, ralenti, inversé et sans horizon, le paysage déstabilise 


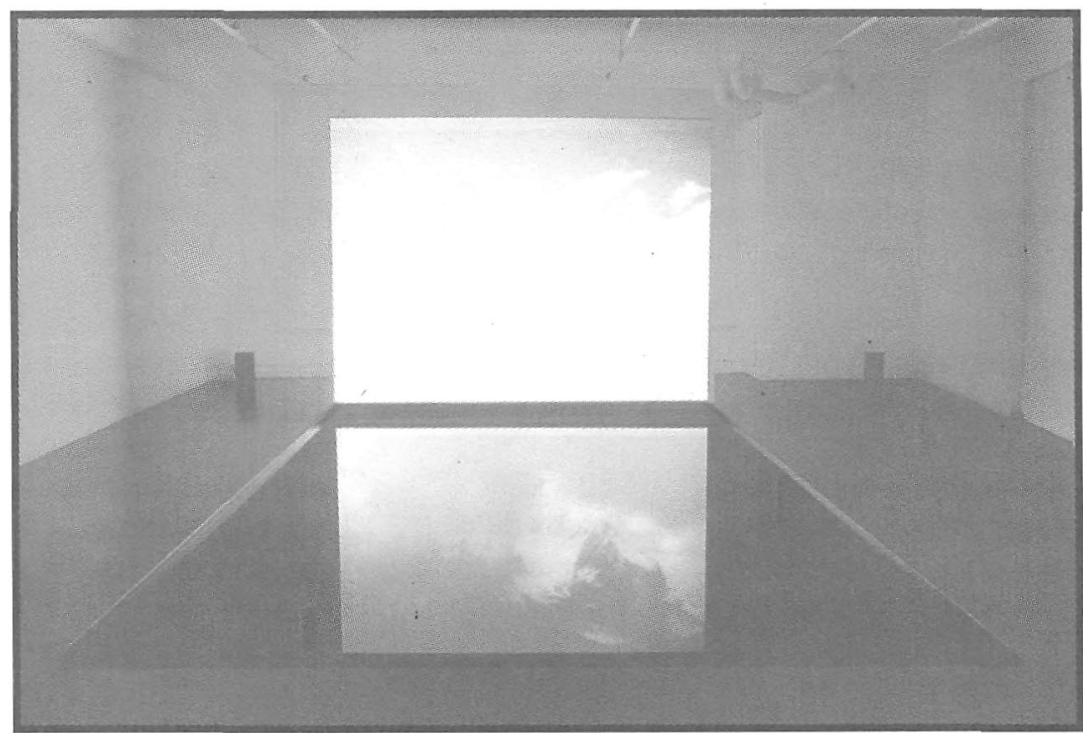

Figure 2

Bertrand R. Pitt, Dérive, 2000-2002, Installation vidéo sonore. Projection vidéo au dessus d'un plan d'eau, équipements audio et vidéo. Dimensions de l'écran : $3 \mathrm{~m} \times 2,25 \mathrm{~m}$, dimensions au sol $6 \mathrm{~m} \times 3 \mathrm{~m}$. Photo: Guy L'Heureux. Espace: Galerie B-312, Montréal, septembre 2002. Gracieuseté de l'artiste.

le regard. Est mise en échec toute tentative d'emprise sur la nature, l'expérience du paysage se faisant plutôt dans le vacillement et la « dérive». Le spectateur est convié à une expérience phénoménologique du paysage. Son installation vidéo plus récente intitulée Tumultes (2003-2004, fig. 3) s'inscrit dans le même esprit donnant à voir, cette fois, un paysage laurentien. L'installation est composée de trois images vidéo projetées sur trois murs différents : un cours d'eau, une main qui écrit puis froisse la page

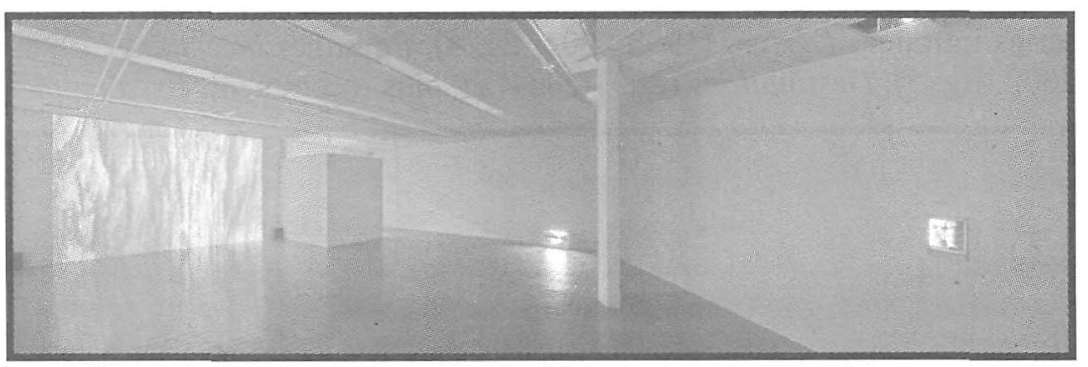

Figure 3

Bertrand R. Pitt, Tumultes, 2003-2004, Installation vidéo sonore à 3 canaux. Projection vidéo au mur (largeur approx. $7 \mathrm{~m}$ ), écran LCD 13", moniteur au sol 21", équipements audio et vidéo. Dimensions variables. Photo : Guy L'Heureux Montage photo: Bertrand R. Pitt. Espace: Plein sud, Longueuil. Gracieuseté de l'artiste. 


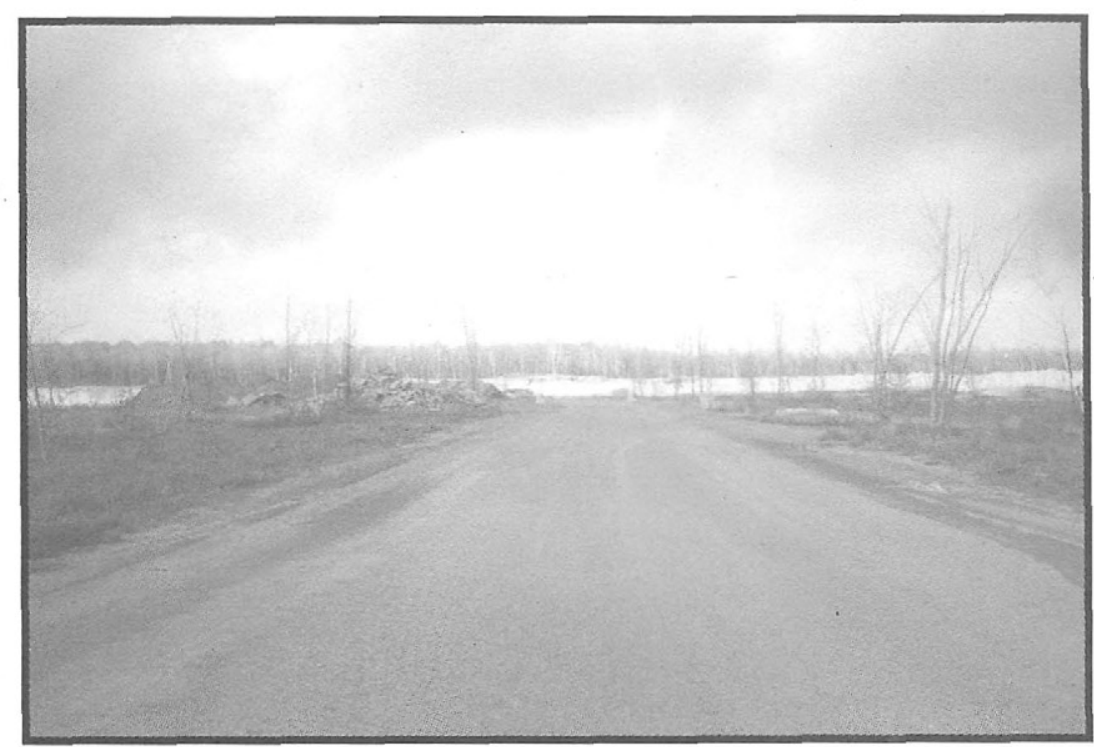

\section{Figure 4}

Isabelle Hayeur, Impasse, (Horizon de sable), 1999, de la série Dérives. Gracieuseté de l'artiste.

raturée et des flammes. Les flammes sont en réalité l'image d'un cours d'eau tumultueux en négatif et coloré en un camaïeu d'orangés. Les crépitements que produisent les flammes ne sont pas ceux de la combustion, mais bien ceux du papier froissé, obtenus en amplifiant les basses fréquences des bruits de rature. Bref, ici encore, les dispositifs d'inversion, de trompe-l'œil, les subterfuges et l'idée de renversement évacuent les possibles structures narratives, détournant du coup la nature laurentienne de sa fonction traditionnelle d'icône nationale québécoise ${ }^{34}$.

Enfin, les grands panoramas photographiques d'Isabelle Hayeur, ses séries intitulées Dérives (1998-2001, fig. 4), Paysages incertains (19982002, fig. 5), Destinations (2003-2004) et, plus récemment, Excavations

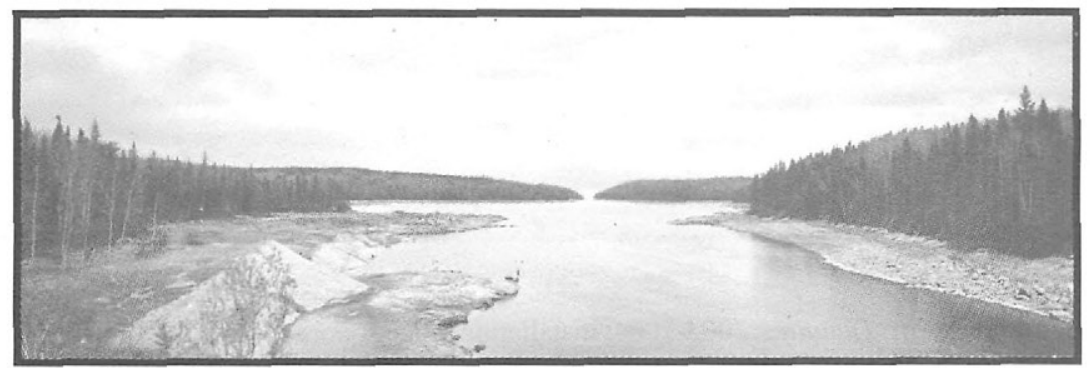

\section{Figure 5}

Isabelle Hayeur, Voie, 2001, de la série Paysages incertains. Gracieuseté de l'artiste. 


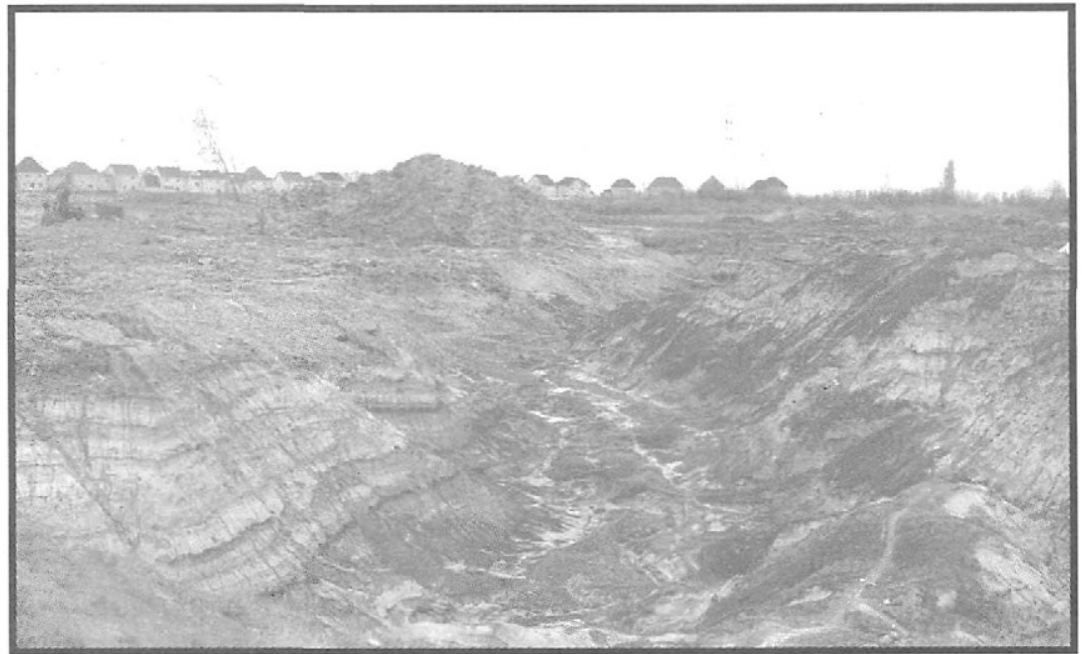

Figure 6

Isabelle Hayeur, Succession, 2005, de la série Excavations. Gracieuseté de l'artiste.

(2005, fig. 6) mettent en scène des espaces, urbains et naturels, contrefaits. Hayeur photographie différents lieux, elle numérise ensuite ces images et les juxtapose afin de former une image en apparence réaliste. Succession, par exemple, fusionne par une inversion d'échelle un paysage ancien, les Badlands, lieux de mémoire, et nouveau, la banlieue construite par des opérations de nivellement, de remplissage et donc par des effacements du paysage naturel et historique. Des espaces référentiels parcellaires et mouvants émergent de ces associations chimériques. Ces paysages hybrides jouent sur l'effet déstabilisant et témoignent de l'éclatement et du morcellement à l'œuvre dans notre rapport contemporain tant au territoire urbain que naturel. Puisqu'ils s'affichent comme simulacres, les montages d'Hayeur insistent sur l'écart entre le signifié et le signifiant et, de ce point de vue, participent à une entreprise de déconstruction et de démystification des conventions esthétiques qui ont façonné le paysage romantique transcendant. Mais encore, le format et la beauté des images continuent d'évoquer l'envoûtement et l'exaltation propres à la tradition romantique qui faisait de la nature une icône de plénitude. La tradition romantique suppose une mise à distance fondée sur la reconnaissance de la nature comme autre, un autre transcendant. Le paysage romantique exprimait justement le sentiment de perte de soi vers un ordre supérieur à celui de la condition humaine. Et au fond, la dérive, l'errance et le débordement ne sont pas étrangers à l'état contemplatif, à un état souhaité de plénitude, de communion. Il existerait donc chez Hayeur une tension dialectique entre l'intensité émotive convoquée et la charge critique de l'œuvre; à la fois un désir de négation et un besoin de continuer à imaginer un certain état de plénitude ${ }^{35}$ ou tout au moins une forme de réconciliation. 
Tout compte fait, la fragilisation, le débordement et la fissuration propres à la déconstruction età la démystification caractérisent nombre de pratiques contemporaines dont le récit continue de s'intéresser au paysage. Pourtant, en dépit d'un certain constat d'échec, les grands espaces ouverts de Pratt, Pitt et Hayeur, pour ne nommer qu'eux, préservent la sublimité propre à la tradition paysagiste romantique. Faisant contrepoids à l'impasse et à la fracturation postmodernes, la dérive et la mouvance peuvent-elles donc aussi être des notions structurantes? Elles m'apparaissent à tout le moins porteuses d'une quête de sens qui contrevient à la radicalisation actuelle de l'individualisme. Ces quelques exemples nous permettent de relever des affinités qui rendent manifeste la persistance d'un désir d'imaginer le paysage - comme le dit si bien en anglais Petra Halkes, «Aspiring to the Landscape " - un espace au sein duquel existent des communautés d'être. C'est aussi ce qui ressort de l'exposition récente Conversations Adru (2004) d'Irène Whittome. L'exposition rassemblait des cuvres issues d'un projet commencé en 1999, à l'époque où l'artiste s'est mise à explorer les carrières désaffectées de la région de Stanstead au Québec tout près de la frontière américaine. Le projet multidisciplinaire visait d'abord à documenter des ruines d'une géologie transformée par l'homme. L'exposition incluait par exemple la photographie d'une usine locale évacuée et aseptisée sur laquelle se superpose une deuxième photographie des travailleurs ayantoccupé l'espace en 1936. La pièce principale, Ellipse 10/19 (2004), était constituée d'une structure de bois formant un ovale ouvert, entourée de photographies panoramiques des vues intérieures de la carrière Adru. Évoquant la brièveté de la vie, l'espace rappelait celui d'une matrice originelle où l'histoire humaine, ses paysages et la nature s'entrelacent. La structure était accompagnée d'une bande sonore qui reproduisait des chants d'oiseaux, le bruit du vent dans les carrières et l'enlèvement des éclats de granit. Il s'agissait, selon Whittome, de reproduire un écho de la démarche créatrice entendue comme " une conversation avec le site (...) une écoute de la pulsation de la terre ".

Transcendant les nationalismes démodés et les sentiments de perte de sens, ce qui semble mis à profit ici, c'est le désir de recréer des filiations, des liens communautaires et de dégager un sens de l'histoire. Il s'agirait alors d'un art faiseur de mythes, mais cette fois de mythes d'origines imaginés à partir d'images archétypales.

Cet article visait à cerner quelques aspects spécifiques du rapport entre la formation d'une identité fondée sur la nationalité et les représentations du paysage dans l'art moderne et postmoderne au Canada. Cet exercice impliquait de caractériser les références au paysage dans l'art actuel en les situant par rapport à un passé récent qui, lui, ancrait le paysage moderne dans un rapport complexe aux idéologies politiques. Sans prétendre à l'exhaustivité, j'ai choisi de commenter des œuvres phares et d'autres 
moins connues, mais qui m'apparaissent non moins symptomatiques de tendances marquantes pour l'une et l'autre des périodes dont il est question.

On convient en général que l'état sauvage et inhabité du territoire canadien tel que mis en scène par le Groupe des sept est devenu rapidement un symbole d'unité nationale au lendemain de la Guerre. En fait, entre 1910 et 1960 , le paysage comme thème pictural - tout comme les œuvres d'art qui évoquent la représentation paysagère - échappe rarement à sa vocation d'objet fétiche capable d'évoquer des sentiments d'unité nationale. À ce sujet, la réception critique des ouvres non figuratives de Borduas datant de 1953 et 1.954 est probante. La persistance de références au paysage (par le format, la gamme chromatique ou le titre) dans ce travail de Borduas fut perçue par plusieurs critiques d'art comme un aspect de sa pratique ancré dans la nationalité. Au-delà de cette interprétation assez simpliste, les références métaphoriques au paysage, tantôt accueillant tantôt sclérosant, peuvent être interprétées comme le signe d'une quête identitaire profondément dichotomique et liée à la condition d'exil de Borduas.

À partir de la fin des années 1960 , plusieurs artistes posent un regard critique sur la valeur symbolique du paysage et de la nationalité. Tout un travail de déconstruction traverse des pratiques aussi diverses que l'art conceptuel, la performance et la peinture, celle de Christopher Pratt par exemple. On ne croit plus à la valeur de la nationalité comme référent organisateur et on conçoit dorénavant le paysage comme un concept culturel où se jouent des luttes de pouvoir. Pour Belmore, Blondeau et Turner, entre autres, il s'agit de se réapproprier par le geste artistique, le passé colonisateur de l'histoire canadienne et de révéler ses marques sur le territoire.

Les notions de déracinement, de dérive et d'exil me sont apparues récurrentes dans les œuvres récentes de Rainville Pitt, Binet et Hayeur. Installations vidéo et photomontages tablent sur la fragmentation et la perte de sens. Ces entreprises de dépaysement, de déstabilisation, d'effacement des limites et des repères ne mènent toutefois pas nécessairement à l'abandon d'une quête identitaire imaginée dans son rapport au lieu et à la nature. Que ce soit par l'entremise d'une expérience du paysage profondément enracinée dans l'expérience sensorielle, chez Rainville Pitt et Binet, ou dans la mémoire et l'histoire, chez Hayeur, ces paysages continuent d'évoquer le sublime; un espace fascinant où il serait possible de rêver une certaine communauté d'être. Kristeva ne disait-elle pas que "l'abject est bordé de sublime ${ }^{36}$." Bien que soient évacués les mythes fondés sur l'unité du territoíre, de la langue et des traditions, j'ai retenu de la notion de nationalité associée au paysage, le désir d'exister dans une relation d'être à la nature, à l'autre, à l'espace environnant. 


\section{Notes}

1. Charles Taylor, Les sources du moi. La formation de l'identité moderne, Montréal, Boréal, 1998, p. 51.

2. Au sujet de la référence au paysage chez Wanda Koop, Stephen Hutchings et Eleonor Bond, on pourra consulter Petra Halkes, Aspiring to the Landscape. On Painting and the Subject of Nature, Toronto, Toronto University Press, 2006.

3. Simon Schamna, Landscape and Memory, Toronto, Random House, 1995, p. 10.

4. W. J. T. Mitchell, (ed.) Landscape and Power, Chicago, University of Chicago, Press, 1994.

5. Joyce Zemans, « Establishing the Canon: Nationhood, Identity and the National Gallery's first Reproduction Program of Canadian Art ", Journal of Canadian Art History, vol. $16 \mathrm{n}^{\circ}$ 2, 1995, p. 6-39.

6. Peter Larisey, Light for a Cold Land: Lawren Harris's Work and Life - An Interpretation, Toronto, Dundurn Press, 1993, p. 60.

7. Lawren Harris, cité dans Charles Hill, L'émergence d'un art national, Ottawa, Musée des beaux-arts du Canada, 1995, p. 89.

8. Michael Tobby, The True North. Canadian Landscape Painting 1896-1939, London, Lund Humphries, Barbican Art Gallery, 1991, p. 42.

9. Charles Hill, L'émergence d'un art national, Ottawa, Musée des beaux-arts du Canada, 1995, p. 75.

10. Newton MacTavish, The Fine Arts in Canada, Toronto, Macmillan Co., 1925, p. 159.

11. Fred Houser, A Canadian Art Movement: The Story of The Group Of Seven, Toronto, Macmillan, 1926.

12. Edith-Ánne Pageot, « Ambiguïtés de la réception critique de l'exposition "Canadian Women Artists", Riverside Museum, New York, 1947 ", RACAR, revue d'art canadienne, vol. 27, $\mathrm{n}^{0} 2,2000$, p. 123-134.

13. François-Marc Gagnon, "La peinture des années trente au Québec », Annales d'histoire de l'art canadien/The Journal of Canadian Art History, vol 3, $\mathrm{n}^{\mathrm{os}} 1-2$, automne 1976, p. 2-20.

14: À ce sujet voir, Laurier Lacroix, Suzor-Côté, lumière et matière, Ottawa, Musée des beaux-arts du Canada, Québec, Musée National des beaux-arts du Québec, 2002, p. 161-223.

15. Lettre citée dans Guy Robert, Marc-Aurèle Fortin, l'homme à l'œuvre, Montréal, Stanké, 1976, p. 121-122.

16. Lettre de Paul-Emile Borduas à Claude Gauvreau, 19 janvier 1959 citée dans François-Marc, Gagnon, Paul-Emile Borduas. Biographie critique et analyse de l'cuvve, Montréal, Fides, 1978, p. 457.

17. André-G. Bourassa et Gilles Lapointe, (éd.), Refus global et autres écrits, Montréal, l'Hexagone, 1997, p. 67.

18. En fait, dès 1934, Borduas rêvait d'un exil à Tahiti ou dans les Nouvelles-Hébrides. François-Marc Gagnon, « La peinture des années trente au Québec ", op. cit., p. 17.

19. Pour connaître les détails qui entourent le séjour de Borduas aux Etats-Unis, on pourra consulter François-Marc Gagnon, Paul-Émile Borduas. Biographie critique et analyse de l'cuvre, Montréal, Fides, 1978, p. 323 et suivantes.

20. La période new-yorkaise de Borduas marque un moment de rupture avec le surréalisme ainsi qu'une prise de conscience des enjeux propres à l'expressionnisme abstrait. À ce sujet voir, François-Marc Gagnon, Paul-Emile 
Borduas, Montréal, Musée des Beaux-Arts de Montréal, 1988, p. 300 et suivantes.

21. Outre le corpus dont il est question ici, Borduas aborde également le paysage à travers le thème de l'île qui apparaît sporadiquement dans sa production entre 1941 et 1958.

22. Carlyle Burrows, « Art: Native and Foreign Features ", The New York Herald Tribune, 24 mars 1957, p. 1. Cité dans François-Marc Gagnon, Paul-Emile Borduas, Montréal, Musée des beaux-arts de Montréal, 1988, p. 316.

23. Suzanne La Brie, L'identité collective des Québécois d'origine canadiennefrançaise, Montréal, Association pour l'éducation culturelle du Québec, 1993, p. 16.

24. François-Edné Rameau, France aux colonies, Paris, A. Jouby Librairie éditeur, 1859 , p. 241.

25. Marcel Rioux, Les Québécois, Paris, Seuil, 1975, p. 15 et Bernard Arcand, Abolissons l'hiver, Montréal, Boréal, 1999, p. 43.

26. R. M. Vaughan, "Tania Kitchell's Winter Wonderland. Exporting Cold Weather and Desire from Toronto to Europe ", Canadian Art, vol. 21, $\mathrm{n}^{\circ} 1$, printemps 2004, p. 41.

27. Georges-Émile Lapalme, Le paradis du pouvoir, mémoires, Montréal, Leméac, coll. «Vie et mémoires », t. III, p. 157. Cité dans Francine Couture, Les arts visuels au Québec dans les années soixante. La reconnaissance de la modernité, Montréal, vlb éditeur, 1993, p. 13.

28. L'auteure interprète le travail de Borduas sur les valeurs de noir et de blanc comme l'expression d'une réconciliation, et non pas d'une dichotomie, entre l'héritage d'une expression autochtone canadienne-française et un sentiment d'appartenance au contexte nord-américain. Louise Vigneault, Identité et modernité dans l'art au Québec. Borduas, Sullivan, Riopelle, Montréal, Hurtubise HMH, 2002, p. 149, 154-155.

29. Écrits I, Édition critiquée par André-G. Bourassa, Jean Fisette et Gilles Lapointe, Montréal, Les Presses de l'Université de Montréal, Université du Québec à Montréal, 1987 , p. 674.

30. Jocelyn Maclure, Récits identitaires. Le Québec à l'épreuve du pluralisme, Montréal, Québec Amérique, 2000, p. 209.

31. À ce sujet voir Craig Calhourn, Nationalism, Buckingham, Open University Press, 1997.

32. Édith-Anne Pageot, " Anatomie du désir. Réflexions sur les configurations de la violence dans l'art de la performance ", Etc Montréal, $\mathrm{n}^{\circ} 67$, sept. oct. nov. 2004, p. 33-37.

33. Bertrand Rainville Pitt, Dérives, Galerie B 312, cahier $n^{\circ} 65,2002$, n.p.

34. Pour une analyse sur le sens symbolique de la nature laurentienne dans l'imaginaire québécois, on pourra consulter Serge Courville, Jean-Claude Robert et Normand Séguin, "Space and Identity: Quebec and the Laurentian Valley ", Canadian Issues/Thèmes canadiens, vol. 20, 1998.

35. Notre examen de cette dimension des œuvres de Hayeur rejoint celle de Petra Halkes à propos des cuvres de Wanda Koop, Stephen Hutchings et Eleonor Bond. Petra Halkes, " Aspiring to landscape », op. cit.

36. Julia Kristeva, Pouvoirs de l'horreur, Paris, Éditions du Seuil, 1980, p. 19. 